\title{
An Air Container Loading Planning Model: DHL and Air Hong Kong Case
}

\author{
Hwa-Joong Kim*, Sang-Won Seo**, Minyoung Park ${ }^{* * *}$, Jae Joon Han****
}

\begin{abstract}
This paper presents a case study on the problem of loading air containers in air express carriers motivated from DHL and Air Hong Kong. The problem is to determine the containers to be loaded and the locations of the loaded containers in an aircraft while maintaining stability of the aircraft. The objective of the problem is to maximize the revenue obtained from delivering containers. We present an integer programming model to represent and optimally solve the problem. Computational experiments done on a number of randomly generated test instances show that the integer program can be a viable tool for generating loading plans in the companies since optimal or near-optimal solutions for the test instances are obtained within a reasonable amount of computation time.
\end{abstract}

Keywords: air express carriers, container loading planning, optimization

Submission Date: 5/03/2011 Revision Date: 05/25/2011 Acceptance Date: 05/25/2011

* Corresponding Author: Professor, Graduate School of Logistics, Inha University, Num-gu, Incheon, 402-751, KOREA. E-mail: hwa-joong.kim@inha.ac.kr, Phone: +82-32-860-8238, Fax: +82-32-860-8223

** Graduate School of Information Management and Security, Korea University, Seongbuk-gu, Seoul, 136-701, KOREA

**** Professor, Graduate School of Logistics, Inha University, Num-gu, Incheon, 402-751, KOREA.

**** Professor, Global Finance and Banking, Inha University, Num-gu, Incheon, 402-751, KOREA. 


\section{Introduction}

Air cargo services can be classified into two categories: express cargo and general cargo services. The general cargo business is still the most commonly used service. However, the past two decades have shown increased growth in the express cargo market. It is estimated that the global air cargo market will grow at an average rate of 5.6\% between 1999 to 2018, while the air express market grew by 22\% between 1994 to 1995 , and the growth rate will increase by $37 \%$ before the year 2015 (Yan et al., 2006). These days, the four largest integrators: Federal Express (FedEx), UPS, DHL, and TNT, account for about $90 \%$ of the world air express service market (Zhang and Zhang, 2002).

This research is motivated from the current practice of DHL and Air Hong Kong. Cathay Pacific recently entered into a business partnership with DHL to provide overnight short-haul cargo services to Asian regional destinations (Zhang and Zhang, 2002). As a result, DHL's express cargo is delivered by Air Hong Kong which is a Cathay Pacific's Hong Kong based sister company. Air Hong Kong now serves Bangkok, Penang, Singapore, Taipei, Narita, Kansai, Nagoya, Incheon, Shanghai and Peking with its Hong Kong hub and spoke system. For the express cargo services of DHL and Air Hong Kong, on-time delivery is a key issue which must take place in order for them to ensure next-day delivery in the Asian market. However, there are many constraints like the late arrival of cargo data and the delayed generation of loading plans which disturbs on-time delivery. Moreover, DHL currently uses a short cut-off time (30 minutes) policy in order to accept additional express cargo from customers. This policy means that there is only a short amount of time available to complete all operations, e.g., developing a loading plan, loading the cargo, and so forth, before the flight's departure. As a result, there are sometimes flight delays.

Among the various issues, this research intends to contribute to the literature and practice by considering the loading planning problem that determines a loading plan for the air containers, pallets and bulks. In real practice, Air Hong Kong is in charge of creating the loading plans for the containers which are received by and packed into the cargo containers by DHL. The current loading plans are developed manually by experienced workers, namely a loadmaster, at Air Hong Kong. The load masters use a trial-and-error approach to satisfy a number of constraints which must be satisfied in a load plan. Of these restrictions, stability is a key constraint since accidents could occur if the constraint is not satisfied in a loading plan. To satisfy the constraint, a lot of time is spent when developing a loading plan in the company. Therefore, this paper considers the loading plan problem while insuring the stability of the aircraft during its take-off, flight, and landing. In real practice, Air Hong 
Kong receives revenue based on the weight of the loaded (delivered) containers and hence, the loadmaster tries to load as much weight as possible onto each plane. Therefore, the objective is to maximize the revenue obtained by loading (delivering) the cargo. To solve this problem, our paper suggests an integer programming model which is a well-known operations research technique and suitable to tackle the problem which Air Hong Kong currently faces.

This paper only reviews articles after 1985 since Martin-Vega (1985) provides a comprehensive review of the literature related to our research up until that year. $\mathrm{Ng}(1992)$ considers the problem of selecting the best loading plan among a number of possible loading plans which are generated in advance while considering priorities of the cargo and the total amount of space that the cargo will occupy. This problem is solved by a multi-criteria goal programming model with the objective of maximizing the cargo load without exceeded the capacity of the aircraft. Amiouny et al. (1992) consider a special case of our problem with the objective of making the center of gravity be as close as possible to a target point. Their heuristic produces a good solution to this problem in terms of quality and time. Later, Marthur (1997) suggests a better heuristic algorithm to deal with the problem considered in Amiouny et al. (1992). Thomas et al. (1998) present a case study of FedEx which uses the same aircraft model which is chosen in our research. To solve the problem, they suggests a heuristic algorithm which consists of two phases. The first phase generates an initial loading plan by using an integer programming model without a specific objective function. The loading plan is later modified by adding constraints to reflect the current load. If the current load is infeasible in terms of the stability constraint, the non-compliant containers are unloaded. This routine continues until a feasible loading plan is obtained. Although this research considers the same problem that we are currently addressing, our research reflects more realistically loading constraints such as aircraft stability restriction and time restriction regarding flight departure. Mongeau and Bes (2003) consider the problem with the objective of maximizing the total weight of the cargo and suggests an integer programming model in order to solve the problem. Their solution considers three stability restrictions: the weight limit for the cargo, the weight limit of location where the containers are loaded, and the center of gravity restrictions. The center-of-gravity is calculated by using a conventional method. That is, the weight of the loaded containers and the weight of the aircraft is calculated and then the weight and the distances between the locations from the aircraft head are multiplied. The multiplication is then summed with the center of gravity of the aircraft without the cargo. Finally, the sum is divided by another sum of the weight of the loaded containers and the weight of the aircraft. However, our research uses a more elaborate method to consider the stability restrictions than the conventional method described above. In addition, time restrictions regarding the 
flight departure is not considered in Mongeau and Bes process (2003). Recently, Yan et al. (2006, 2008) consider a fairly general planning problem in a hub-and-spoke system when different destinations are desired for the cargo. Delivering containers to different locations often results in repackaging of some containers having to take place in a hub, and stochastic demand at the destinations. However, the stability restrictions and amount of loading time are not considered in their research due to the complexity of these matters.

This paper is organized as follows. The next section specifically defines our problem as well as describing the amount of cargo space on the aircraft, the type of containers, and the stability restrictions. An integer programming model is presented in Section 3 based on the specified problem. Section 4 summarizes the data collection and generation method and Section 5 follows with an explanation of the test results. Finally, we summarize our research results and suggest future research directions in Section 6.

\section{Problem description}

This section describes the amount of aircraft cargo space and the container and pallet types which are considered in this research as well as addressing the stability restrictions which must be followed in order to ensure the safe take-off, flight, and landing of the aircraft.

\subsection{Aircraft cargo space and container and pallet types}

Figure 1 presents the amount of cargo space on the aircraft which we have chosen for this research. The model is an Airbus A300-600, which is the primary aircraft used by Air Hong Kong. As you can see in Figure 1(a), the cargo space of this aircraft is divided into an upper deck and a lower deck according to the deck's longitude, and each deck consists of a number of locations where containers, pallets and bulks are loaded, e.g., 16 locations in the upper deck and 10 locations in the lower deck in Figure 1(a). There are three doors where containers can enter and exit the airplane: a door for the upper deck is located in the rear of the aircraft and two doors for the lower deck are located in the rear and front of the plane in Figure 1(a). Zone is a set of locations in which the direction and location of the downward forces are the same, i.e., zone A consists of locations 1 and 17, zone $\mathrm{K}$ consists of locations 11 and 24, and zone $\mathrm{O}$ consists of location 15 in Figure 1(b). In Figure 1(b) on the cross sections of the aircraft, the left half of the aircraft is called the left lane and the right half is referred to as the right lane. 


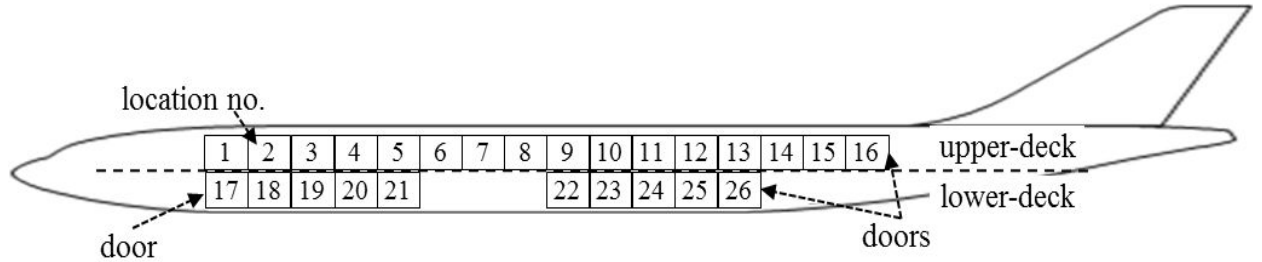

(a) Vertical section

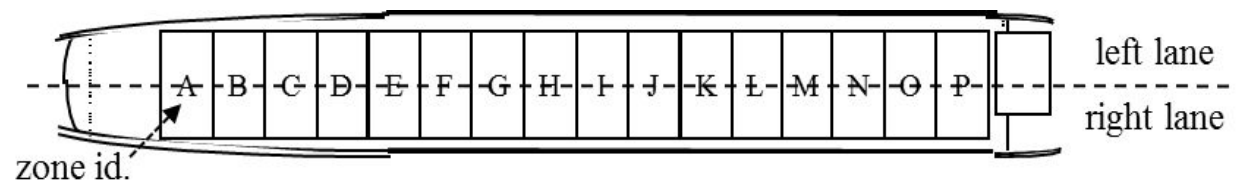

(b) Cross section

\section{Figure 1.}

Cargo space of an Airbus A300-600

There are a number of container types and pallet types that can be loaded onto the aircraft. Overall, they can be classified into single-row types and side-by-side types. One container (pallet) of the single-row type can be loaded into that location while two containers (pallets) of the side-by-side type can be loaded in that location. This implies that if a container (pallet) of the single-row type is loaded into a location, other containers (pallets) cannot be loaded in the same location while a container (pallet) of the side-by-side type is loaded in a location, only a containers (pallets) of the same type can be loaded into the same location. In order to consider this concept in our model, we assume that a location is composed of three different positions for modeling purposes. In Figure 2, the dots represent positions (a location consists of three positions as can be seen in Figure 2) and the numbers in parenthesis represent the position numbers. Side-by-side containers can only be loaded into positions next to walls in each location, e.g., postions (1), (3), (4), and (6) in Figure 2 while the single-row type containers can be loaded only into the center positions, e.g, positions (2) and (5) in Figure 2. If a side-by-side type container is loaded into position (1), position (2) will not be available for other containers. However, position (3) is available only for side-by-side containers. On the contrary, if a single-row type container is loaded into position (5), none of the containers can be loaded into the other points at the same location. Here, let the adjacent points be other points in the same location where any container cannot be loaded if that position is occupied by another container. Bulk can only be loaded into the right side location of the lower deck, i.e., location 26 in Figure 1(a). 
Case 1: a side-by-side type container is loaded

Case 2: a single-row type container is loaded

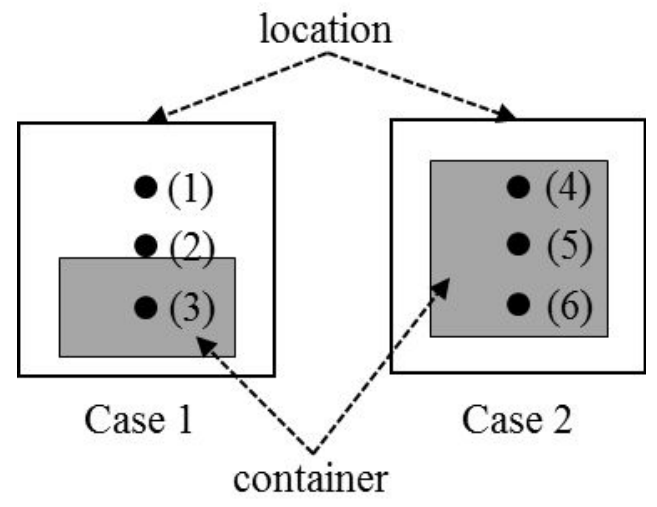

Figure 2.

Location occupied by each container type

Each container (pallet) type is different in size. There are nine container types which can be used in the aircraft: type 1 (single row container 88" x 125"), type 2 (single row container 96" x 125"), type 3 (single row container 125" x 96"), type 4 (side-by-side container $125^{\prime \prime}$ x 88"), type 5 (side-by-side 1 container $25^{\prime \prime}$ x 96"), type 6 (side-by-side container LD3), type 7 (single row pallet 88" x 125"), type 8 (single row pallet 96" x 125"), and type 9 (single row bulk). Here, 96" x 125" implies the length and height of a container which is 96 inches and 125 inches, respectively. Types 1, 2, 3, 4, 5 can only be loaded into the upper deck of the aircraft, while types $6,7,8,9$ can only be loaded into the lower deck. Type 4 can also only be loaded like a single row type container into locations 13, 15,16 due to space limitations in this area even though it is a side-by-side type container. Note that even though the lengths of some types of containers are longer than the zone's lengths, this is considered by limiting the locations assigned to corresponding containers and also, during real loading operations, the locations can be adjusted by considering the gaps between the adjacent containers, which is done in real practice by Air Hong Kong. Table 1 shows the possibility of loading each container type into each location. For example, type 1 can be loaded into all of the locations in the upper deck except for location 16. However, type 3 can only be loaded into locations $1,3,4,5,7,8,10$. 


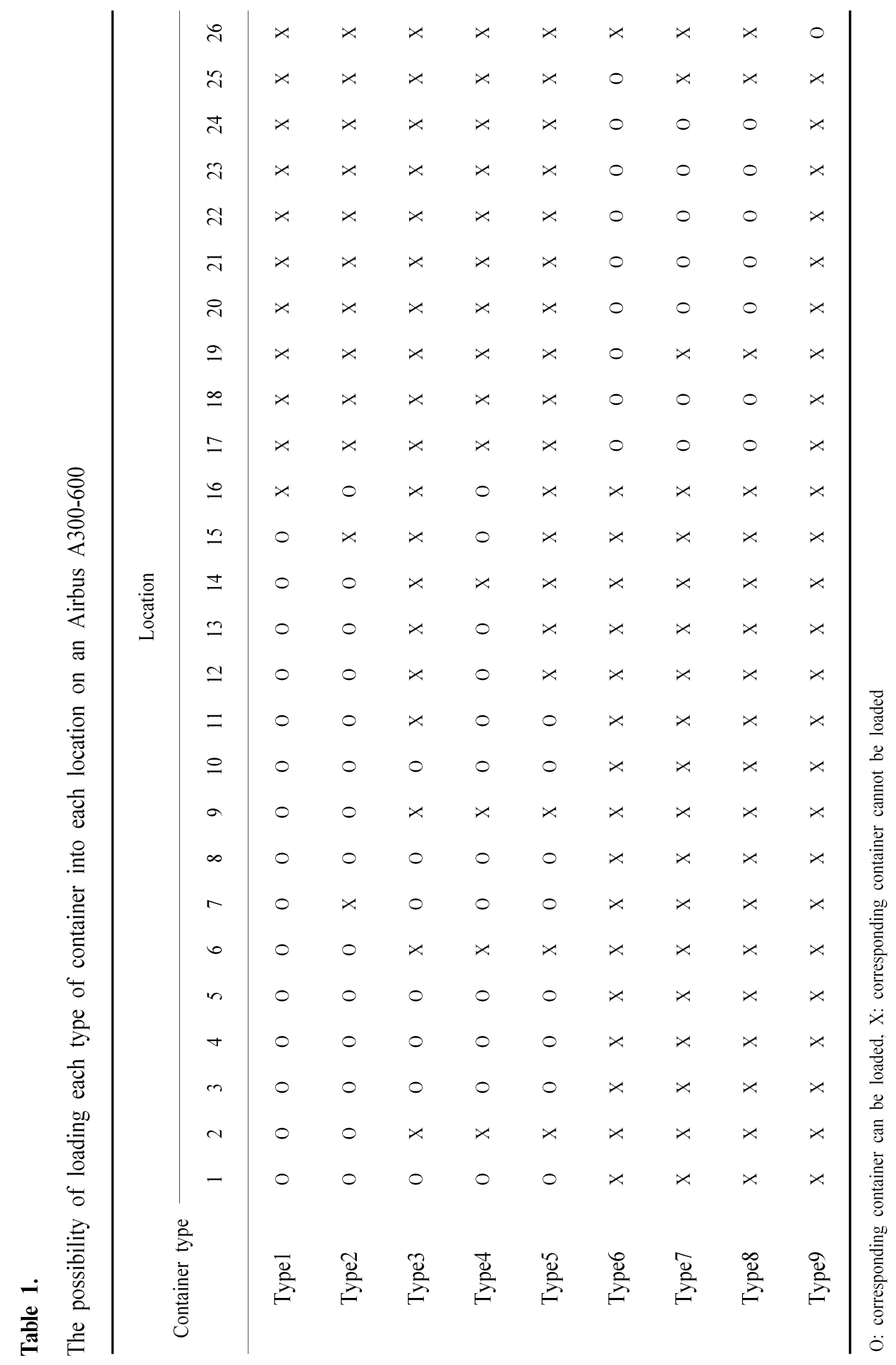




\subsection{Stability restrictions}

There are four types of stability restrictions of the aircraft considered in this research: the cumulative load limit of each zone; the location load limit of each location and container (pallet) type and bulk cargo; the lateral load imbalance limit; and a center-of-gravity restriction. These limits are essential in order to ensure a safe take-off, flight, and landing.

Cumulative load limit. Each zone has an individual cumulative load limit. To illustrate the limit, we will first define the different zones within one zone. Previous zones are zones located on left side or right side of the zone. The distintion between the left and right side is zone $\mathrm{E}$ in the case of A300-600 in Figure 1(b). For example, the previous zones of zone $\mathrm{E}$ are zones $\mathrm{A}, \mathrm{B}, \mathrm{C}$, and $\mathrm{D}$, while those of zone $\mathrm{F}$ are zones $\mathrm{G}, \mathrm{H}, \mathrm{I}, \mathrm{J}, \mathrm{K}, \mathrm{L}, \mathrm{M}, \mathrm{N}, \mathrm{O}$, and $\mathrm{P}$ in Figure 1(b). The cumulative load limit of a zone implies that the total weight of all of the cargo in the previous zones and itself should not be more than the limit of the zone. For example, the total weight of all of the cargo loaded in zones A, B, C, D, E should not be more than the cumulative load limit of zone E in Figure 1(b). The cumulative load limit of zone $\mathrm{E}$ is $19,877 \mathrm{~kg}$ in Table 2(a) which shows the cumulative load limit of the Airbus A300-600.

Location load limit. The load limit implies that the weight of the cargoes loaded into a location should not be more than the weight limit of that location. The load limit is different depending on the location and the types of containers being used. For example, if the container type is type 2 and they are loaded into location 3, the load limit is $3,696 \mathrm{~kg}$. However, as shown in Table 2(b), the limit is $5,670 \mathrm{~kg}$ if the same containers are loaded into location 6. 


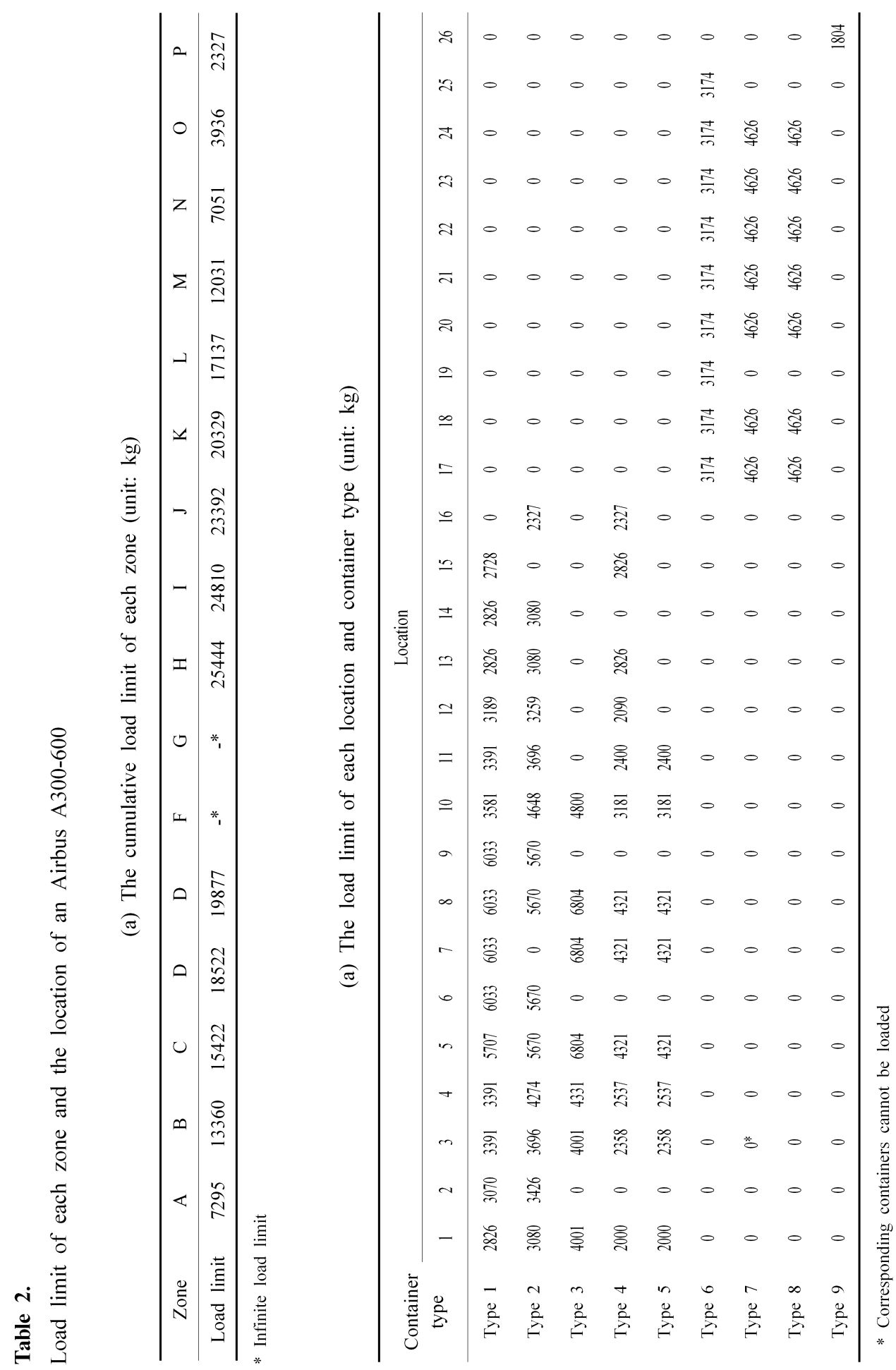


Lateral load imbalance limit. When side-by-side containers (pallets) are loaded, special attention must be paid to the lateral loading difference. The weight difference between left and right lanes of the aircraft must not exceed the imbalance limit. However, upper and lower decks have different imbalance limits, i.e., the limit of the upper deck is $6,000 \mathrm{~kg}$ while that of the lower deck is $1000 \mathrm{~kg}$ in the case of the Airbus A300-600.

Center-of-gravity restriction. This restriction is intended to maintain the longitudinal stability during the take-off, in-flight, and landing of an aircraft. The restriction is based on the total index units and total weight of the load on the aircraft. The index unit is a measure related to the load weight in each location. Table 3 shows the real data of the index unit for the Airbus A300-600. For example, the index unit for location 4 is -73 if the weight of the containers in this location is 2000 from Table 3. Based on the real data in Table 3, an equation consisting of the index unit and load weight can be obtained using a simple regression analysis like the one shown here.

$$
I_{l}=b_{l} w_{l}+c_{l} \quad \text { for } \quad l \in L
$$

where $I_{l}$ is the index unit for location, $w_{l}$ is the load weight in that location $l, b_{l}$, and $c_{l}$ are factors for location $l$ which are obtained by the regression analysis, and $L$ is the set of locations. Then, two total index units and two total weights must be considered in order to check the satisfaction of the center-of-gravity restriction. The two total index units (two total weights) are different with respect to whether the weight of the fuel is summed or not when calculating the total index unit (the total weight). The mathematical definitions of the two total index units and two total weights are shown below.

$$
\begin{gathered}
T_{1}=\sum_{l \in L} I_{l}+I_{e}+I_{f}, T_{2}=\sum_{l \in L} I_{l}+I_{e}, \\
T W_{1}=\sum_{l \in L} w_{l}+w e+w f, \text { and } T W_{w}=\sum_{l \in L} w_{l}+w e
\end{gathered}
$$

where we is the weight of the crew and the aircraft without any containers loaded, $I_{e}$ is the index unit corresponding to we, wf is the fuel weight, and $I_{f}$ is the index unit corresponding to $w f$. For simplicity sake, let zero-fuel be the state where the fuel weight is not considered into the calculation and let fuel state be the state where the fuel weight is considered into the calculation. That is, $T I_{1}$ and $T W_{1}$ correspond to the fuel state and $T I_{2}$ and $T W_{2}$ correspond to the zero-fuel state. 


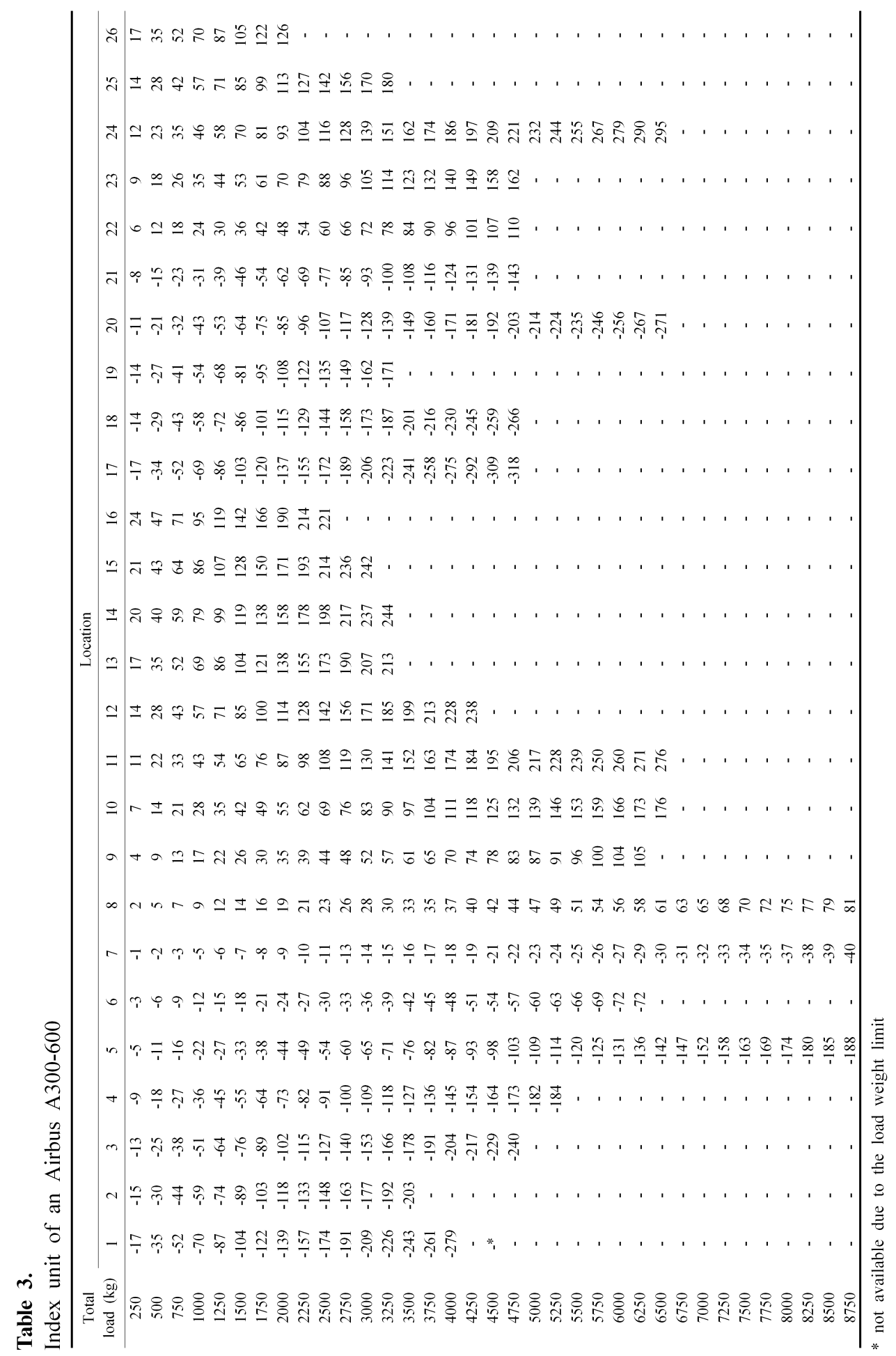


The center of gravity of the aircraft is obtained by using the center-of-gravity envelope in Figure 3 in a real situation. The shaded and slashed areas are the envelopes in Figure 3. There are two envelopes, one is for the zero-fuel state and the other is for the fuel state. In Figure 3, envelope 1 is the envelope for the fuel state and envelope 2 is the envelope for the zero-fuel state. Envelope 1 consists of lines e1, e2, $\cdots$, e8 and envelope 2 consists of lines e1, e9, e10, e5, e6, e7, and e8. That is, the slashed area in envelope 1 includes envelope 2 and the darkened area which can be seen in Figure 3. Envelopes 1 and 1 can be represented by:

$$
\begin{aligned}
& u_{e} T I_{k}+v_{e} \geq T W_{k} \quad \text { for } k \in\{1,2\} \text { and } e \in E M_{k} \\
& u_{e} T I_{k}+v_{e} \leq T W_{k} \quad \text { for } k \in\{1,2\} \text { and } e \in E L_{k}
\end{aligned}
$$

In these formulas, $u e$ and $v e$ are the slope and constant of line $e$ in the center- of- gravity envelopes. Indices 1 and 2 correspond to the envelopes for the fuel state and the zero-fuel state. $E M_{k}$ is set of lines in top of the center-of-gravity envelope $k$, e.g. line e9 if envelope $k$ is envelope 2 in Figure 3 and $E L_{k}$ is a set of lines in the center-of-gravity envelope $k$, except for the top lines in the envelope, e.g., lines e1, e4, e5, e6, e7, e8 if envelope $k$ is envelope 1 in Figure 3. An aircraft's flight is safe, i.e., corresponding loading plan is feasible, when the two points made up of the total index units and total weights, $\left(T L_{1}\right.$, $\left.T W_{1}\right)$ and $\left(T I_{2}, T W_{2}\right)$, are in envelopes 1 and 2, respectively. For example, if the two points are $\mathrm{p} 1$ for the zero-fuel state and $\mathrm{p} 3$ for the fuel state, it is infeasible since $\mathrm{p} 1$ is outside of envelope 2. It is infeasible if the zero-fuel state and the fuel state points are $\mathrm{p} 2$ and $\mathrm{p} 4$ since $\mathrm{p} 4$ is outside of envelope 1. A feasible combination of points in Figure 3 is $\mathrm{p} 2$ and $\mathrm{p} 3$ since $\mathrm{p} 2$ is in envelope 2 and $\mathrm{p} 3$ is in envelope 1. 


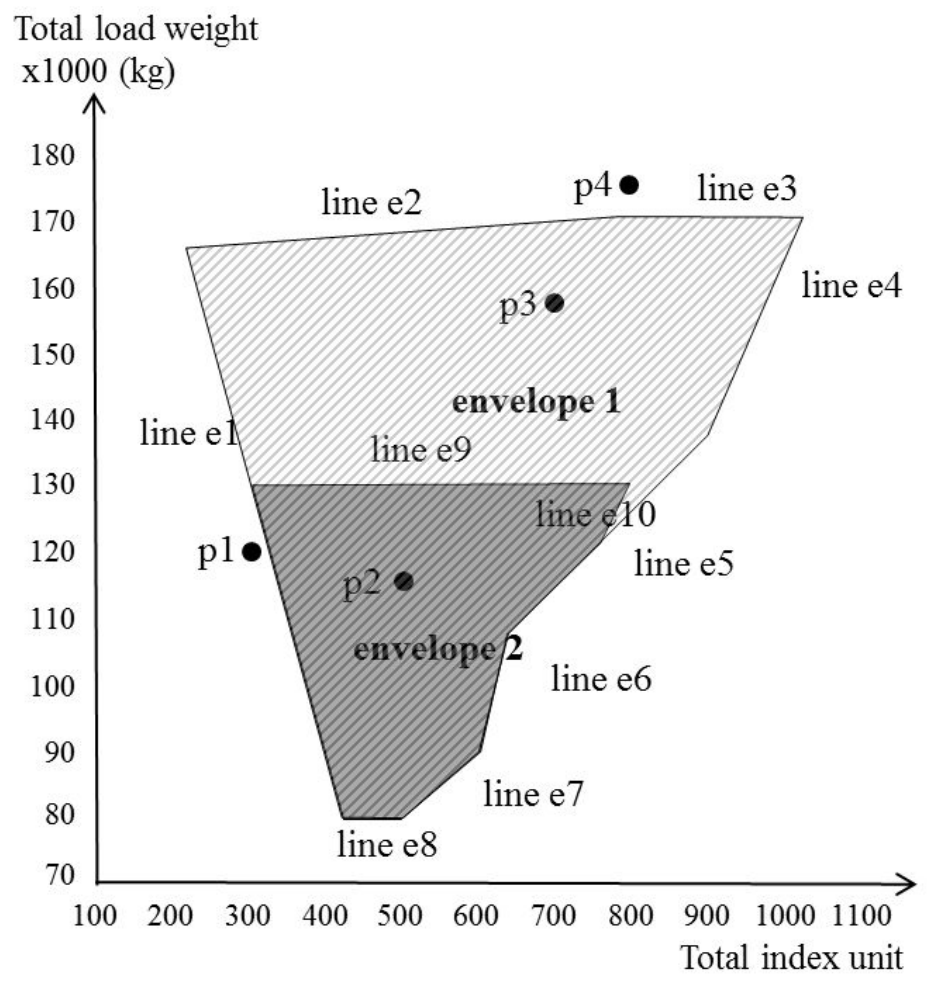

Figure 3.

Center-of-gravity envelope of an Airbus A300-600

\subsection{Problem definition and assumptions}

Now, the problem considered in this research is defined as follows: the problem is to determine the locations for the containers, pallets, and bulk while satisfying the stability restrictions and finishing all of the loading operations before the aircraft is scheduled to depart. The objective of the problem is to maximize the revenue obtained by loading the cargo. This paper considers the containers, pallets, and bulk which arrives at different times. It is assumed that the loading time of the containers, pallets, and bulk is dependent of the loading point according to the real practice in Air Hong Kong. A loading operation for a container can be performed after finishing operations for previous operations far from the door than the position where the container is loaded. All loading operations should also be completed before the departure time of the aircraft in order to avoid delays. On-time delivery is very important for express cargo service companies like DHL where the cut-off time for the cargo is very close to the departure time in order for the maximum amount of 
cargo to be transported. We also assume that any container is not be loaded onto the aircraft before generating the loading plan, i.e., the aircraft is empty. However, one can simply consider the already loaded containers by leaving them in their current locations, i.e., the locations are fixed for these containers. In this case, the locations of the containers that have already been loaded into the cargo spaces far away from the doors must not be considered when loading the newly available containers. Finally, we summarizes the other assumptions which are made in this research:

(a) All data is deterministic and is made available before solving the problem;

(b) The reshuffling of containers is not allowed, i.e., the relocation of containers which were previously positioned is not allowed;

(c) The repackaging of containers is not allowed;

(d) There is not any available idle time between the two loading operations. However, if the idle time is constant, the idle time can be considered by adding the time into the time required to load the containers

(e) All loading operations are successful, i.e., no failure of loading occurs.

\section{An integer programming model}

Before describing the model, we summarize the notations used in the model below. Although containers, pallets, and bulk are considered in this research, they are all referred to by using one single term, i.e., container, here after for simplicity sake.

Sets

$B_{z} \quad$ set of previous locations in zones before zone $z$

$C$ set of containers

$C_{j} \quad$ set of containers that can be located in any position $j$

$E M_{k}$ set of top lines in the center-of-gravity envelope $k$

$E L_{k}$ set of lines in the center-of-gravity envelope $k$, except for the top lines in the envelope

$L \quad$ set of locations

$L_{l} \quad$ set of positions in location $l$ 
$L E_{d}$ set of positions in the left lane of deck $d$

$R I_{d} \quad$ set of positions in the right lane of deck $d$

$P \quad$ set of positions

$P_{i} \quad$ set of positions where container $i$ can be loaded

$Q_{j} \quad$ set of positions where other containers cannot be loaded if a container is loaded into position $j$

$T$ set of container types

$T_{t} \quad$ set of containers in container type $t$

$Z \quad$ set of zones

$Z_{z} \quad$ set of locations in zone $z$

\section{Parameters}

$a_{i} \quad$ arrival time of container $i$

$b_{l} \quad$ slope of location lin the corresponding regression equation

$c_{l} \quad$ intercept of location lin the corresponding regression equation

$c_{t} \quad$ closing time of the aircraft

$c w l_{z}$ cumulative weight limit for zone $z$

$f_{j} \quad$ position that corresponds to the loading operation which should be finished before the loading operation for position $j$ since it is farther away from the door than position $j$

$I_{e} \quad$ index unit for the weight of the crew and the aircraft without any loaded containers

$I_{f} \quad$ index unit for the fuel weight

$r_{i} \quad$ revenue of container $i$

$t_{j} \quad$ loading time of position $j$

$u_{e} \quad$ slope of line $e$ in the center-of-gravity envelopes

$v_{e} \quad$ constant of line $e$ in the center-of-gravity envelopes

$w_{i} \quad$ weight of container $i$

$w b_{d}$ limit of the weight imbalance (left and right) of deck $d \in\{1,2\}$ where 1 and 2 represent the upper and lower decks of the aircraft, respectively

we weight of crew and the aircraft without any loaded containers

$w f \quad$ weight of the fuel

$w l_{t l} \quad$ weight limit of container type $t$ and location $l$

$|\cdot|$ size of set $\bullet$ 


\section{Decision variables}

$T I_{k}$ total index unit of state $k \in\{1,2\}$ where 1 and 2 represent a zero-fuel state and a fuel state, respectively

$T W_{k}$ total load weight unit of state $k$

$X_{i j} \quad=1$ if container $i$ is loaded into position $j$, and 0 otherwise

$Y_{j} \quad$ total loading time of position $j$

The integer programming model suggested in this research is presented below:

Maximize $\sum_{i \in C j \in P_{i}} r_{k} X_{i j}$

subject to

$$
\begin{aligned}
& \sum_{j \in P_{i}} X_{i j} \leq 1 \\
& \text { for } i \in C \\
& \sum_{i \in C_{j}} X_{i j} \leq 1 \\
& \text { for } j \in P \\
& \sum_{h \in Q_{j} \in C_{h}} X_{g h} \leq\left|Q_{j}\right|\left(1-X_{i j}\right) \\
& \text { for } i \in C \text { and } j \in P_{i} \\
& \sum_{j \in L_{l} i \in C_{j} \cap T_{t}} w_{i} X_{i j} \leq w l_{t l} \\
& \sum_{l \in Z_{z} \cup B_{z} \in L_{l} i \in C_{j}} \sum_{i} X_{i j} \leq c w l_{z} \\
& \sum_{j \in L E_{d} i \in C_{j}} \sum_{i} X_{i j}-\sum_{j \in R I_{d} i \in C_{j}} \sum_{i} X_{i j} \geq-w b_{d} \quad \text { for } d \in\{1,2\} \\
& \sum_{j \in L E_{d} i \in C_{j}} \sum_{i} X_{i j}-\sum_{j \in R I_{d} i \in C_{j}} w_{i} X_{i j} \leq w b_{d} \quad \text { for } d \in\{1,2\} \\
& \sum_{j \in L}\left(b_{l} \sum_{j \in L_{l} i \in C_{j}} w_{i} X_{i j}+c_{l}\right)+I_{e}+I_{f}=T I_{1} \\
& \sum_{j \in L}\left(b_{l} \sum_{j \in L_{l}} \sum_{i \in C_{j}} w_{i} X_{i j}+c_{l}\right)+I_{e}=T I_{2} \\
& \sum_{i \in C j \in P_{i}} w_{i} X_{i j}+w e+w f=T W_{1} \\
& \sum_{i \in C j \in P_{i}} w_{i} X_{i j}+w e=T W_{2} \\
& u_{e} T I_{k}+v_{e} \geq T W_{k} \\
& \text { for } t \in T \text { and } l \in L \\
& \text { for } z \in Z
\end{aligned}
$$




$$
\begin{array}{ll}
u_{e} T I_{k}+v_{e} \leq T W_{k} & \text { for } k \in\{1,2\} \text { and } e \in E L_{k} \\
Y_{j} \leq \sum_{i \in C_{j}}\left(a_{i}+t_{j}\right) X_{i j} & \text { for } j \in P \\
Y_{j} \geq Y_{f_{j}}+t_{j} \sum_{i \in C_{j}} X_{i j} & \text { for } j \in P \\
Y_{j} \leq c t & \text { for } j \in P \\
X_{i j} \in\{0,1\} & \text { for } i \in C \text { and } j \in P_{i} \\
Y_{j} \geq 0 & \text { for } j \in P \\
T I_{k}, T W_{k} \geq 0 & \text { for } k \in\{1,2\}
\end{array}
$$

The objective function of this problem is to maximize the total revenue of the loaded containers. Constraint (4) implies that each container can only be loaded onto the plane once, while Constraint (5) implies that each position can only be occupied by one container. Constraint (6) implies that if a container is loaded into a location, none of the other containers can be loaded into adjacent positions in the same location where the first term is the number of loaded containers on the adjacent positions in the same location. That is, $\sum_{h \in Q_{g} \in C_{h}} X_{g h}=0$ if $X_{i j}=1$. The stability restrictions are represented in constraints (7) (16). Constraints (7) and (8) imply the load limit of a location and the load limit of a zone, respectively. Constraints (9) and (10) both represent the lateral imbalance restriction of each deck and describe the restriction in the format of the integer programming model though the restriction has an absolute value as follows:

$$
\left\|\sum_{j \in L E_{d} i \in C_{j}} \sum_{i} X_{i j}-\sum_{j \in R I_{d} i \in C_{j}} w_{i} X_{i j}\right\| \leq-w b_{d}
$$

where $\|\cdot\|$ is the absolute value of $\bullet$. Constraints (11) - (16) represent the center-of-gravity restriction. Constraints (11) - (14) are equations for calculating the total index unit and the total weight of the loaded containers and the center-of-gravity envelopes are represented by Constraints (15) and (16). Constraint (17) implies that the loading completion time of a position is calculated by considering the arrival time of the container to be loaded into a position and the amount of time required for the loading to take place. Constraint (18) implies that the loading completion time of a position should be after the sum of the time of a position just before the position and the loading time of the position. Constraint (19) implies that all of the loading operations should be completed before the 
departure time of the aircraft. Finally, constraints (20) - (22) are restrictions on the decision variables.

\section{Data collection and generation}

The data which was required for running the integer programming model was collected from Figures 1, 2, 3, and Tables 1, 2, and 3. This data was randomly generated based on the advice of a manager at Cathay Pacific. There are two types of data: data related to the aircraft and data related to the containers.

All data related to the aircraft was obtained from Figures 1, 2, 3, and Tables 1, 2, 3 except for the index unit for the weight of the crew and the aircraft without any containers loaded, the index unit and the weight of the fuel, the lateral imbalance limit, the parameters in equations (1), (2), and (3), the loading time, departure time and closing time. The weight of the crew and the aircraft without any containers loaded was set to $82,479 \mathrm{~kg}$. This was obtained from real data and the corresponding index unit is 302 , the fuel weight was set to $27,000 \mathrm{~kg}$ and the corresponding index unit is 32 from a preliminary calculation. The lateral imbalance limits of the upper and lower decks were set to $6,000 \mathrm{~kg}$ and $1000 \mathrm{~kg}$ as previously stated. The parameters $b_{l}$ and $c_{l}$ in equation(1) and $u_{e}$ and $v_{e}$ in constraints (2) and (3) are summarized in Table 4 and were obtained by a preliminary calculation. As you can see in Table 4(a), the regression equation explains very well the relationship between the index units and the load weight of each location since all $R$-square values are nearly one. The loading time of the positions which are close to the doors were set at five minutes while the loading time for location $i$ was increased by 0.1 minutes from the loading time of location $j$ just before location $i$ in terms of the closeness to the door. Five minutes is very close to the time required for lifting a container up to the door and the slight difference $(0.1$ minutes) between the loading times of location $i$ and $j$ is due to the movement of the container in the cargo space since the container must be rolled into a location after being lifted up to the door of the cargo space. The flight departure time and the closing time were set at noon ( 720 minutes $=12 \cdot 60)$, and five minutes before noon (715 minutes), respectively, for modeling purposes. Five minutes is required after the closing time in order for take-off-related work to be performed. 


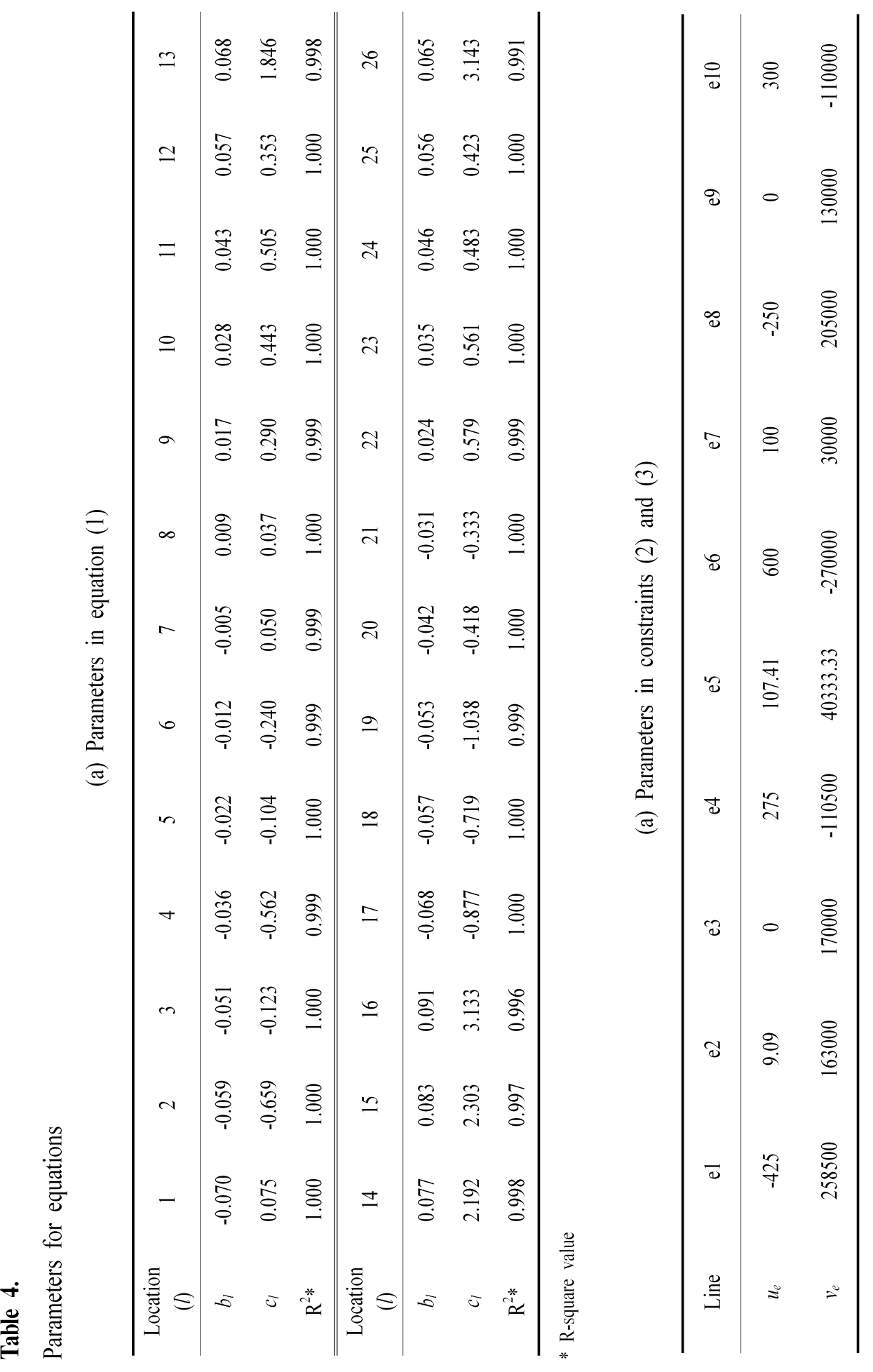


Now, we will describe our methods of generating data related to the containers such as the container type, weight, revenue, and arrival time of each container. The container type is set to container types $1,2, \cdots, 9$ with probabilities of $0.05,0,05,0.1,0.3,0.3,0.1,0.05$, $0.01,0.01$, respectively. The weight of a container (unit: $\mathrm{kg}$ ) is randomly generated using $U(1000,6300), U(1000,5670), U(1000,6804), U(1000,4321), U(1000,4321), U(1000$, $3174), U(1000,4626), U(1000,4626)$, and $U(500,1804)$ for container types $1,2, \cdots, 9$, respectively, where $U(a, b)$ is the uniform distribution with a range of $[a, b]$. The revenue of each container is assumed to be proportional to the weight of the container and hence the revenue per $\mathrm{kg}$ is set to $\$ 1$ U.S. The remaining information focuses on the arrival time of each container.

The arrival times of the containers are assumed to follow the exponential distribution, i.e., the time gap between the arrival times of two containers (inter-arrival time) is exponentially distributed. The mean inter-arrival time was set differently according to the different time zones since $60 \%$ of the containers arrive 4 hours to 1 hour before the departure time, and $40 \%$ arrive thirty minutes before the departure time. Information regarding the average arrival times of the containers was obtained from a manager in the company. For simplicity sake, we made the 4 to 1 hour advanced arrival time represent time zone 1 and the 30 minute advanced arrival time represent time zone 2 . In order to represent these different percentages of arrivals, we use the following equation for time zone $t$ :

$$
\lambda_{t}=\frac{p_{t}}{t d_{f}} N
$$

where $\lambda_{t}$ is the averaged number of the arriving containers per minute in time zone $t$, i.e., a reciprocal number of the mean inter-arrival time in time zone, $p_{t}$ is the arrival probability in time zone $t, t d_{t}$ is the time duration in time zone $t$, and $N$ represents the number of containers. Because of the arrival of containers with different probabilities in different time zones, we set the arrival probability and the time duration of each time zone as follows:

$$
\left\{p_{1}, p_{2}\right\}=\{0.6,0.4\} \text { and }\left\{t d_{1}, t d_{2}\right\}=\{3 \cdot 60,0.5 \cdot 60\} \text { minutes. }
$$

Also, the starting time in each time zone is set to $\left\{s_{1}, s_{2}\right\}=\{8 \cdot 60,11 \cdot 60\}$ minutes since the flight departure time is 720 minutes and the starting time of each time zone is 4 hours. We must also add 1 hour to the flight departure time for time zones 1 and 2, respectively. Therefore, the arrival time of each container is obtained by using the following procedure: 
Step 1. For each time zone, we set

$$
Z N_{t}=0 \text { for } t=1,2 .
$$

where $Z N_{t}$ is the number of containers assigned to time zonet.

Step 2. Set $i=1$.

Step 3. The time zone of container $i$ is randomly selected among three times zones with the probabilities $1-p$ and $p$ for time zones 1 and 2 , respectively ( $p$ is 0.4 in real practice).

Step 4. If $Z N_{t}=0$, set the arrival time of container $i\left(a_{i}\right)$ to be the starting time of time zone $t\left(s_{i}\right)$ and them move to Step 5. Otherwise, the inter-arrival time of container $i\left(T_{i}\right)$ is obtained by using the inverse function of cumulative probability functions of exponential distribution:

$$
T_{i}=-\frac{1}{\lambda_{t}} \ln \left(1-P_{i}\right)
$$

where $P_{i}$ is the probability that the waiting time for container $i$ is not more than the mean inter-arrival time of container $\lambda_{t}$. We randomly generated probability $P_{i}$ using $U(0,1)$ and the arrival time of container $i$ is set to

$$
a_{i}=a_{\psi(i)}+T_{i}
$$

where $\psi_{i}$ is the container that arrives just before container $i$.

Step 5. Set $Z N_{t}=Z N_{t}+1$ and $i=i+1$. If $i>N$, stop and otherwise, go to Step3.

\section{Test results}

To demonstrate the performance of this integer programming model, computational tests were performed on various test instances. Firstly, an output example of the model was given to graphically illustrate the output. Secondly, we ran more general tests on randomly generated container data. Finally, the effects of different arrival time patterns were analyzed. The model was also tested using CPLEX 11.2, a commercial software program which we ran on a personal computer with a Pentium processor at an operating speed of $1.73 \mathrm{GHz}$. During the tests, the CPLEX was terminated every time that the computation time reached 1200 seconds. In this case, the percentage deviation from the lower bound was used as the performance measure. Note that the percentage deviations can be obtained 
directly from the CPLEX. The performance measures used are the number of optimal solutions which were obtained by the CPLEX, the percentage deviation from the lower bound (for the cases where the CPLEX cannot give the optimal solutions within 1200 seconds), and the computation time. The time limit (1200 seconds) was obtained from the actual time that it usually takes for the generation of a loading plan to be set up by a loadmaster at Air Hong Kong, i.e., it takes an average of 20 minutes for a loadmaster at Air Hong Kong to generate a feasible loading plan if all of the needed data is available.

Figure 4 shows an example of a loading plan which was obtained by the model when 40 containers were onboard the plane. The objective function value of the model is $\$ 47,519.2$ U.S., and the computation time took only 0.73 seconds. Hence, this loading plan is optimal since the computation time took less than 1200 seconds (the time limit for the CPLEX). Fourteen containers among the available containers were selected for loading and the numbers of each container type were:

$$
\left\{N_{1}, N_{2}, N_{3}, N_{4}, N_{5}, N_{6}, N_{7}, N_{8}, N_{9}\right\}=\{2,2,0,3,4,4,0,0,0\}
$$

where $N_{t}$ is the number of containers in container type $t$ to be loaded. Table 5 shows the feasibility of the loading plan in Figure 4. As can be seen in Table 5, the cumulative load limit restriction is satisfied since all of the cumulative loads in all of the zones are less than the limit. The lateral imbalance limit restriction is also satisfied since the imbalances in the lower and the upper decks are 3554.2 and $367.4 \mathrm{~kg}$, which are less than the acceptable imbalance limits of the decks. The total weights and indices are 156,998.2 $\mathrm{kg}$ and 573.5 respectively for envelope 1 , and $129,998.2 \mathrm{~kg}$ and $541.5 \mathrm{~kg}$ for envelope 2 . Therefore, the corresponding points are in the envelopes and can be known from Figure 3. 
Objective functional value: 47,519.2 US\$

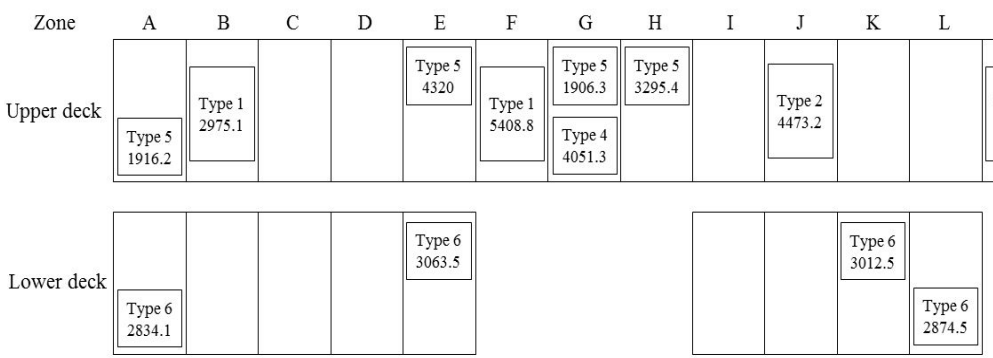

Figure 4.

A loading plan model

Table 5.

The feasibility of a loading plan by the model

(a) The cumulative load limit of each zone (unit: $\mathrm{kg}$ )

\begin{tabular}{cccccccccccccccccc}
\hline Zone & A & B & C & D & E & F & G & H & I & J & K & L & M & N & 0 & P \\
\hline $\begin{array}{c}\text { Cumulative } \\
\text { weight }\end{array}$ & 4750.3 & 7725.4 & 7725.4 & 7725.4 & 15108.9 & - & - & 21043.9 & 17748.5 & 17748.5 & 13275.3 & 10262.8 & 7388.3 & 4777.8 & 2534.6 & 0 \\
$\begin{array}{c}\text { Cumulative } \\
\text { weight limit }\end{array}$ & 7295 & 13360 & 15422 & 18522 & 19877 & $-*$ & $-*$ & 25444 & 24810 & 23392 & 20329 & 17137 & 12031 & 7051 & 3936 & 2327 \\
\hline
\end{tabular}

(b) The lateral imbalance limit of each deck (unit: $\mathrm{kg}$ )

\begin{tabular}{ccccc}
\hline & Left lane & Righ lane & Imbalance & Imbalance limit \\
\hline Upper deck & 9521.7 & 5967.5 & 3554.2 & 6000 \\
Lower deck & 6076 & 5708.6 & 367.4 & 1000 \\
\hline
\end{tabular}

(c) Total weight and index

\begin{tabular}{ccc}
\hline & Total weight & Total index \\
\hline Envelope 1 & $156,998.2 \mathrm{~kg}$ & 573.5 \\
Envelope 2 & $129,998.2 \mathrm{~kg}$ & 541.5 \\
\hline
\end{tabular}


In order to run a general test of the integer programming model, we randomly generated 50 test instances, i.e., 10 instances for five levels of the number of containers $(20$, $30,40,50$, and 60). The test results are summarized in Table 6 which shows the numbers of the optimal solutions (obtained from the CPLEX) and the percentage deviations from the lower bounds (when the CPLEX could not give an optimal solution within 1200 seconds), and the computation time. As can be seen from the table, all test instances were solved optimally except for two test instances which contained 20 containers. The average computation time was less than 6 seconds except for the 2 test instances which had 20 containers. However, the computation time for the test instances with 20 containers are still significantly less than 20 minutes, which is how long it normally takes the loadmasters at Air Hong Kong to generate a feasible loading plan. Therefore, the integer programming model is very useful from a practical perspective.

\section{Table 6.}

Performance of the integer programming model on different numbers of containers

\begin{tabular}{c|c|c|c}
\hline $\begin{array}{c}\text { Number of } \\
\text { containers }\end{array}$ & Nopt* $^{*}$ & Gap ${ }^{\dagger}$ & $\begin{array}{c}\text { Computation time } \\
\text { (seconds) }\end{array}$ \\
\hline \hline 20 & 8 & 0.00 & 314.19 \\
30 & 10 & 0.00 & 5.50 \\
40 & 10 & 0.00 & 3.30 \\
50 & 10 & 0.00 & 3.07 \\
60 & 10 & 0.00 & 3.10 \\
\hline
\end{tabular}

* Number of problems (out of 10 problems) that gave the optimal solutions

† Percentage deviation from the lower bound (averaged over 10 problems). Here, 0.00 implies that the average percentage deviation was less than $0.005 \%$

† Average computation time

In order to show the effects of different arrival times, we used six different probabilities of containers' arrivals during the last thirty minutes $(0.2,0.3,0.4,0.5,0.6$, and $0.7)$. For the test, we generated 60 test instances with 40 containers, i.e., 10 instances for six levels of arrival probabilities. The test results are summarized in Table 7, which also shows that all of the test instances were solved in 6 seconds. Although the computation time seems to increase as the probability increases, it is difficult to conclude that there is a trend for the computation time to increase due to the different probabilities of arrivals. This implies that the integer programming model could generate optimal loading plans within a short computation time even when a majority of the containers arrive during the last thirty minutes. 


\section{Table 7.}

Performance of the integer programming model on different arrival probabilities

\begin{tabular}{c|c|c|c}
\hline Probability & Nopt* & Gap* & $\begin{array}{c}\text { Computation time } \\
\text { (seconds)* }\end{array}$ \\
\hline \hline 0.2 & 10 & 0.00 & 2.13 \\
0.3 & 10 & 0.00 & 2.02 \\
0.4 & 10 & 0.00 & 4.36 \\
0.5 & 10 & 0.00 & 2.63 \\
0.6 & 10 & 0.00 & 5.08 \\
0.7 & 10 & 0.00 & 4.51 \\
\hline
\end{tabular}

* See the footnote in Table 6

\section{Concluding remarks}

This paper considered a planning problem of loading air containers onto airplanes for delivery which is encountered when DHL and Air Hong Kong load. To ensure a safe flight, the problem determines the correct way to load the cargo containers into the aircraft. The objective of the problem is to maximize the revenue which is obtained from loading and delivering the containers. In this research, we presented an integer programming model which attempts to solve this problem. Computational experiments were performed on the data which was collected and randomly generated based on real data. The test results showed that the integer programming model gave optimal or near-optimal solutions for the test instances in a reasonable amount of computation time. Therefore, the integer programming model is a viable tool for generating loading plans at Air Hong Kong in a practical sense. Although our model was developed based on the Airbus A300-600 data, we could extend our model and apply it to other aircrafts in the near future.

This research can be extended in several ways. First, the relocation of the containers which have already been loaded into the aircraft should be considered since more containers could possibly be loaded onto the plane by relocating some of these containers. Second, it is worthwhile to consider the uncertainty of the weight and the arrival time of the cargo is highly uncertain until the cut-off time in real situations. Third, overall performance of the loading plans may be improved by changing the weight of the containers. This research can be then extended by incorporating both the airplane loading plan and the container packaging problems. Fourth, the problem of some cargo moving in different 
directions is worth considering. Finally, if some containers can be loaded onto later flights while still making their delivery dates, the company may be able to generate additional revenue by making better use of the containers on the current flight. However, this case may cause a situation where some containers cannot be loaded onto a later flight due to a shortage of containers. Therefore, in order for this case to be considered, we need to investigate a multi-airplane loading system, which is one of important future research topics. 


\section{Acknowledgement}

This work was supported by the Korea Research Foundation Grant funded by the Korean Government (KRF-2008-005-J01601). Authors would like to express our sincerest gratitude to Mr. Jung-Won Park at Cathay Pacific for giving us quality advice and sharing important information about the daily practices at Air Hong Kong and DHL.

\section{References}

Amiouny, S., J. Bartholdi, J. Vate, and J. Zhang. 1992. Balanced loading, Operations Research, vol. 40, pp. 238-246.

Martin-Vega, L.A. 1985. Aircraft load planning and the computer: description and review, Computers\&Industrial Engineering, vol. 4, pp. 357-369.

Marthur, K. 1997. An integer-programming-based heuristic for the balanced loading problem, Operations Research Letters, vol. 22, pp. 19-25.

Mongeau, M. and C. Bes, 2003. Optimization of aircraft container loading, IEEE Transactions on Aerospace and Electronic Systems, vol. 39, pp. 140-150.

Ng, K.Y.K. 1992. A multicriteria optimization approach to aircraft loading, Operations Research, vol. 40, pp. 1200-1205.

Thomas, C., K. Campbell, G. Hines, and M. Racer. 1998. Airbus packing at Federal Express, Interfaces, vol. 28, pp. 21-30.

Yan, S., C.-T. Lo, and Y.-L. Shih. 2006. Cargo container loading plan model and solution method for international air express carriers, Transportation Planning and Technology, vol. 29, pp. 445-407.

Yan, S., Y.-L. Shih, and F.-Y. Shiao. 2008. Optimal cargo container loading plans under stochastic demands for air express carriers, Transportation Research Part E, vol. 44, pp. 555-575.

Zhang, A. and Y. Zhang, 2002. Issues on liberalization of air cargo services in international aviation. Journal of Air Transport Management, vol. 8, pp. 275-287. 
